\title{
Renal azotemia and associated clinical and laboratory findings in dogs with Babesia rossi infection
}

\author{
P. Defauwa*, S. Daminet ${ }^{a}$, A.L. Leisewitz ${ }^{b}$, A. Goddard ${ }^{b}$, D. Paepe ${ }^{a}$, L. Duchateauc, J.P. Schoeman ${ }^{b}$ \\ a Small Animal Department, Faculty of Veterinary Medicine, Ghent University, Salisburylaan 133, 9820 Merelbeke, Belgium \\ b Department of Companion Animal Clinical Studies, Faculty of Veterinary Science, University of Pretoria, Pretoria, Onderstepoort 0110 , \\ South Africa \\ ${ }^{c}$ Biometrics Research Group, Faculty of Veterinary Medicine, Ghent University, Salisburylaan 133, 9820 Merelbeke, Belgium
}

*Corresponding author.

Tel No.: +3292647700; Fax No.: +3292647791; E-mail address: pieter.defauw@ugent.be (P. Defauw)

Small Animal Department, Faculty of Veterinary Medicine, Ghent University, Salisburylaan 133, 9820 Merelbeke, Belgium

This work was partially presented as a research report at the 2017 ACVIM forum, National Harbor, MD.

Highlights

- Renal azotemia was diagnosed at presentation in $14 \%$ of all dogs.

- Based on urine specific gravity, renal azotemia was an underdiagnosed event.

- Based on urine osmolality, most azotemic dogs had renal azotemia.

- Old age and hyperphosphatemia could help to identify concomitant renal azotemia.

Abbreviations:

AKI, acute kidney injury; ARDS, acute respiratory distress syndrome; ARF, acute renal failure; CKD, chronic kidney disease; IMHA, immunemediated hemolytic anemia; OVAH, Onderstepoort Veterinary Academic Hospital; RA, renal azotemia; RLB, reverse line blot; SIRS, systemic inflammatory response syndrome; USG, urine specific gravity 
The occurrence of acute kidney injury in canine babesiosis is not well documented. Furthermore, interpretation of urine specific gravity (USG) to assess renal concentrating ability is hampered by the frequent presence of hemoglobinuria in this disease.

This cross-sectional study aimed to test the hypothesis that renal azotemia (RA) is underdiagnosed according to current canine babesiosis literature by determining its occurrence at presentation, using urine osmolality instead of USG to measure urinary concentration. The second objective was to examine potential associations between the presence of RA and selected clinical and laboratory variables at presentation. Medical records available from 3 previously performed prospective data collections were reviewed retrospectively. Clientowned dogs that were diagnosed with babesiosis caused by Babesia rossi, were included if a complete blood count, biochemistry profile, and urinalysis was performed at admission. Urine osmolality was measured to identify dogs with RA. Differences between dogs with RA and dogs without RA were assessed by nonparametric statistics.

One hundred and fifty-two dogs were included, of which 26 (17\%) were azotemic at admission. The occurrence of RA was 14\% (21/152), hence $81 \%$ (21/26) of all azotemic dogs were diagnosed with RA. In contrast, when diagnosis of RA was based on an admission USG $<1.030$, only $23 \%$ (6/26) of the azotemic dogs would have been considered to have RA. Several signalment and clinicopathological findings were found to be associated with the presence of RA, including older age, and the presence of collapse, hypoglycemia, hyperphosphatemia, cerebral babesiosis, and acute respiratory distress syndrome. Lastly, survival at discharge was significantly lower in dogs diagnosed with RA at presentation.

Our results clarified that RA is more common than previously reported in B. rossi. This study also demonstrated that USG determination is not a reliable method to evaluate renal concentrating ability in azotemic dogs with babesiosis. Thus, if available, urine osmolality should be part of the diagnostic work-up of dogs infected with B. rossi to avoid misclassification of dogs with RA as having prerenal azotemia. If urine osmolality cannot be measured, clinicians should realize that most azotemic dogs with B. rossi infection have RA.

Keywords:

Acute kidney injury

Babesia rossi

Dogs

Urine osmolality

Urine specific gravity 


\section{Introduction}

Canine babesiosis is an intra-erythrocytic protozoan disease of worldwide clinical importance, which can be caused by several large and small Babesia species (Irwin, 2010). The most prevalent canine Babesia species in South Africa is B. rossi, although infections with B. vogeli have also been detected in dogs in South Africa (Matjila et al., 2004). Babesia rossi infections are associated with a variety of complications, which are thought to be consequences of systemic inflammatory response syndrome (SIRS) that is present in the majority of clinical infections (Welzl et al., 2001; Köster et al., 2015). Although acute kidney injury (AKI) is a recognized complication of canine babesiosis (Lobetti and Jacobson, 2001; Welzl et al., 2001), the occurrence of AKI and acute renal failure (ARF) varies widely in literature pertaining to canine babesiosis, mostly due to highly variable study populations, the presence of sampling biases, and different criteria used to define AKI and ARF. Solely based on studies where no sampling bias was identified, the reported occurrence of azotemia ranges from 0 to $36 \%$ when all Babesia species are included (Lobetti and Jacobson, 2001; Camacho et al., 2004; Furlanello et al., 2005; Ruiz de Gopegui et al., 2007; Zygner and Wedrychowicz, 2009). For B. rossi infections specifically, 13\% of all dogs were azotemic in 1 study (Lobetti and Jacobson, 2001).

The markers traditionally used to detect decreased kidney function, serum creatinine and urea, are generally insensitive markers for early detection of kidney injury and renal dysfunction (Braun et al., 2003). Moreover, the presence of hemolysis in babesiosis leads to several additional limitations in the traditional clinical evaluation of renal function. Because of non-renal causes of an increased serum urea in babesiosis, serum urea has several limitations when used to assess renal function in this disease (de Scally et al., 2006). The presence of elevated serum bilirubin and free hemoglobin can lead to interference and consequently an underestimation of serum creatinine concentrations (de Scally et al., 2004). This interference can occur when serum creatinine is measured using the Jaffe reaction or enzymatic methods, which are the 2 most commonly used analytical techniques for the measurement of creatinine in dogs (Braun et al., 2003; de Scally et al., 2004). Additionally, a previous study documented decreasing plasma creatinine concentrations after experimental B. canis infections, which was suggested to be caused by an increased plasma volume after infection (Schetters et al., 2009). Lastly, urine specific gravity (USG) can be disproportionately increased in the presence of large molecules such as hemoglobin (Chadha et al., 2001; Imran et al., 2010). Evaluation of USG could therefore lead to an overestimation of urinary concentration in canine babesiosis, caused by hemolysisinduced prerenal proteinuria.

Based on these babesiosis-specific limitations to evaluate renal function when using serum creatinine and USG, we hypothesize that renal azotemia (RA) may be underdiagnosed in the current literature on canine babesiosis. Therefore, our primary objective was to determine the occurrence of RA at presentation in dogs with babesiosis caused by B. rossi, using the gold standard method of estimating urinary concentration (i.e., urine osmolality). Our second objective was to explore potential associations between the presence of RA and selected clinical and laboratory variables at presentation.

\section{Material and Methods}

\subsection{Study population}

In this cross-sectional study, 297 medical records from client-owned dogs with babesiosis that presented to the Onderstepoort Veterinary Academic Hospital (OVAH), University of Pretoria, South Africa, were reviewed retrospectively. All records were available from 3 previously performed prospective data collections in 2006 (cohort 1, 89 dogs), 2011-2013 (cohort 2, 100 dogs), and 2013 -2015 (cohort 3, 
108 dogs). Several studies were published originating from the first 2 cohorts (Schoeman and Herrtage, 2007; Schoeman et al., 2007b; Rees and Schoeman, 2008; Schoeman and Herrtage, 2008; Goddard et al., 2015; Köster et al., 2015; Goddard et al., 2016). These studies were all approved by the University of Pretoria's Animal Ethics Committee (V V070-05; V055-11; V034-14).

All dogs that presented with babesiosis during the study periods were evaluated for inclusion, irrespective of disease severity. Diagnosis of babesiosis was initially made by demonstration of the intra-erythrocytic parasite on stained blood smears, and the species was later confirmed as B. rossi by PCR and reverse line blot (RLB) in all dogs. PCR and RLB were also used to exclude dogs with $B$. vogeli and/or concurrent Ehrlichia canis infections. Dogs were also excluded if any obvious wounds or trauma was evident on physical examination, or when concurrent neoplastic, inflammatory, cardiac disease, or other infections were diagnosed. Based on the initial inclusion during the previously performed prospective studies (exclusion when known concurrent disease was identified) and based on a thorough retrospective revision of all medical records, dogs with suspected chronic kidney disease (CKD) were excluded. Whenever a chronic history of weight loss, partial anorexia and/or polyuria/polydipsia was mentioned in the history, dogs were excluded from this study. Dogs known to have been treated with anti-inflammatory medication within 4 weeks prior to presentation were also excluded.

\subsection{Sample collection and laboratory methods}

Blood and urine samples were collected prior to any therapy, especially fluid therapy, was initiated. A complete medical record, including history, results of physical examination, CBC (Cell-Dyn 3700, Abbott Laboratories, Abbott Park, IL, USA; ADVIA 2120, Siemens, Munich, Germany) and serum biochemistry was available at admission for all dogs. Blood pressure was determined by an oscillometric technique. Blood lactate was measured with a hand-held point-of-care lactate device (Lactate Pro, Arkray, Kyoto, Japan). Different biochemistry analyzers were used for cohort 1 (NExCT/VetEX Alfa Wassermann, Bayer, Isando, South Africa) and cohort 2/3 (Cobas Integra 400 plus, Roche Diagnostics, Mannheim, Germany). Serum creatinine was determined using a modified Jaffe method in all dogs, but the reference interval of serum creatinine was different between cohort $1(40-133 \mu \mathrm{mol} / \mathrm{L})$ and cohort $2 / 3(59-109 \mu \mathrm{mol} / \mathrm{L})$. Serum hemoglobin was only measured in cohort 1, using the same biochemistry analyzer. Serum cortisol (Radioimmunoassay cortisol, Coat-A-Count, Diagnostic Products Corporation, CA, USA) and total thyroxine (Radioimmunoassay canine T4, Coat-A-Count, Diagnostic Products Corporation, CA, USA) assays were performed using kits previously validated for dogs (Schoeman et al., 2007b). Dogs were only included in this study if urinalysis was performed at presentation. Most urine samples were collected by cystocentesis, but occasionally samples were obtained by free catch or by catheterization. Urinalysis consisted of a dipstick analysis, microscopic sediment analysis, and USG which was measured by refractometry. In 2016-2017, stored urine samples were used to measure urine osmolality by freezing point depression (MicroOsmometer Autocal Type 13/13DR, Roebling, Berlin, Germany). Measuring urine osmolality with this freezing point osmometer is based on the principle that a solution of $1 \mathrm{Osmol} / \mathrm{kg}$ freezes at a temperature $1.86^{\circ} \mathrm{C}$ lower than that of pure water (Chadha et al., 2001). Before running the samples, osmolality of a calibration standard solution was measured in triplicate every day. All samples were measured in duplicate or triplicate, and the mean urine osmolality and intra-assay coefficient of variation were calculated. The calculated mean values of all urine osmolality measurements from an individual dog were used to determine the presence or absence of RA, since the individual coefficient of variation values were all $<1 \%$ and the global intra-assay coefficient of variation was $0.19 \%$. 


\subsection{Diagnosis of $R A$}

Dogs were divided into 2 groups, based on the presence or absence of RA at admission. Renal azotemia was defined as the presence of azotemia (i.e., serum creatinine concentration above the reference interval) combined with a urine osmolality $<1110 \mathrm{mOsmol} / \mathrm{kg}$ (i.e., the equivalent of USG $<1.030$ ). The conversion of USG $<1.030$ to urine osmolality $<1110 \mathrm{mOsmol} / \mathrm{kg}$ was based on a previously described conversion formula (Ayoub et al., 2013). Azotemic dogs with inappropriately concentrated urine (i.e. USG <1.030) either have intrinsic renal insufficiency (i.e. RA) or an underlying urine concentrating defect (Cowgill and Langston, 2011). Based on the study design, most diseases and medications leading to an impaired urine concentrating ability could be excluded. Because of the negative bias of severe hyperbilirubinemia on serum creatinine concentrations when measured using the Jaffe reaction (de Scally et al., 2004), dogs with severe hyperbilirubinemia and a serum creatinine concentration close to the upper reference value were excluded to avoid potential misclassification of azotemia. Hyperbilirubinemic dogs were excluded only if the total bilirubin concentration exceeded $85 \mu \mathrm{mol} / \mathrm{L}$, which is the concentration at which significant interference $(> \pm 10 \%$ of the initial serum creatinine concentration) may occur according to the manual of the chemistry analyzers (Glick et al., 1986), in combination with a serum creatinine concentration close ( $\pm 10 \%$ ) to the upper reference limit.

\subsection{Clinical assessment}

Presence or absence of SIRS was based on canine-specific criteria, as previously described and applied in canine babesiosis (Okano et al., 2002; Köster et al., 2015). Defining uncomplicated disease and complications were based on previously published criteria for complicated babesiosis and severe falciparum malaria (Jacobson and Clark, 1994; Jacobson, 2006; WHO, 2014). Dogs were considered to be collapsed at presentation when they were unable to walk unaided. Hypotension was defined as a mean arterial pressure (MAP) $<80$ mmHg. Severe anemia was diagnosed when the hematocrit was $<0.15$. Hemoconcentration was defined as a hematocrit $>0.37$ in combination with signs of intravascular hemolysis (macroscopic hemoglobinuria and/or hemoglobinemia). Secondary immune-mediated hemolytic anemia (IMHA) was diagnosed based on a positive in-saline agglutination test. Hypoglycemia was diagnosed when blood glucose was $<3.3 \mathrm{mmol} / \mathrm{L}$. Jaundice was diagnosed when severe hyperbilirubinemia was present (total bilirubin $>50 \mu \mathrm{mol} / \mathrm{L}$ ). Cerebral babesiosis was defined as the presence of neurological signs that could not be attributed to any other cause, such as hypoglycemia. Acute respiratory distress syndrome (ARDS) was suspected clinically based on the presence of dyspnea and variably based on arterial blood gas analysis, radiological evidence of pulmonary edema, and exclusion of other causes of pulmonary edema. All dogs received standard antibabesial treatment with diminazene aceturate (Berenil RTU, Intervet, Kempton Park, South Africa) at $3.5 \mathrm{mg} / \mathrm{kg}$, and supportive care such as packed red blood cells and intravenous fluid therapy as needed. Any complications were treated accordingly at the discretion of the attending clinician. Outcome categories were survival (i.e., short-term survival until discharge) or death/euthanasia due to poor prognosis.

\subsection{Statistical analysis}

A commercial software program was used for all statistical analyses (SAS version 9.4, SAS Institute Inc., Cary, NC, USA). Differences between dogs with RA and dogs without RA were assessed by the Wilcoxon rank-sum test for continuous and ordinal categorical variables, while the Fisher's exact test was used for binary variables. Clinical variables that were compared included age, body weight, duration of illness, body temperature, heart rate, respiratory rate, and mean arterial pressure. Hematological and biochemical variables included the 
hematocrit, white blood cell count, segmented and band neutrophil count, platelet count, serum creatinine and urea, glucose, total bilirubin, cortisol, total thyroxine, blood lactate, and serum hemoglobin. Variables from the urinalysis included pH, protein, bilirubin, blood/hemoglobin, color, turbidity, USG, and urine osmolality. Binary or ordinal variables originating from clinical, clinicopathological data and the presence or absence of specific complications were also compared. These variables included the presence of collapse, hypotension, severe anemia, absence of anemia, presence of SIRS, hyperphosphatemia, hyperlactatemia, IMHA, hemoconcentration, jaundice, hypoglycemia, cerebral babesiosis, ARDS, the presence of $\geq 1$ other complication, and outcome at discharge. Level of significance was set at $5 \%(P$-value $<0.05)$.

\section{Results}

\subsection{Study population and occurrence of AKI, azotemia, and RA}

Medical records from 297 dogs infected with B. rossi were evaluated for inclusion. One dog with severe hyperbilirubinemia (total bilirubin of $163 \mu \mathrm{mol} / \mathrm{L}$ ) and a serum creatinine concentration close to the upper reference limit (serum creatinine of $104 \mu \mathrm{mol} / \mathrm{L}$ ) was excluded to avoid potential misclassification of azotemia. Azotemia was present at admission in 15\% (44/296) of the initial dog population.

Since urinalysis was not performed at admission in 144 dogs, only 152 dogs were finally included in the study. Azotemia was present at admission in 17\% (26/152) of the final study population. The occurrence of RA at presentation was 14\% (21/152) among all dogs included, hence present in $81 \%(21 / 26)$ of the azotemic dogs. In contrast, when RA was defined as the presence of azotemia combined with a USG $<1.030$, only $4 \%(6 / 152)$ of all dogs included and only $23 \%(6 / 26)$ of azotemic dogs would have been considered to have RA. The relationship between the USG and urine osmolality of each dog with RA is visualized in Fig. 1.

\subsection{Comparison between dogs with and without renal azotemia}

In the group of 21 dogs with RA, there were 9 Boerboel (43\%), 3 Rottweiler (14\%), and 2 mixed breed dogs (10\%), while 16 Boerboel (12\%), 2 Rottweiler (2\%), and 28 mixed breed dogs (21\%) were present in the 131 dogs without RA. Several other dog breeds were represented by a single dog in the RA group. Data from dogs with and without RA are summarized and compared in Tables 1-4. Dogs with RA were significantly older than dogs without RA. In Fig. 2, age distribution is presented in histograms, demonstrating a right-skewed distribution in dogs without RA (Fig. 2A), in contrast to a bimodal distribution in dogs with RA (Fig. 2B). Dogs with RA had a significantly higher body weight and lower body temperature. Presence of collapse was significantly associated with the presence of RA, while gender, duration of illness, and mean arterial pressure were not. Most hematological parameters were not significantly different between both groups, except for band neutrophil count, which was significantly higher in dogs with RA. Serum urea, total bilirubin, serum hemoglobin, and lactate concentrations were significantly higher in dogs with RA. Hyperphosphatemia and hypoglycemia were significantly more common in dogs presenting with RA, while the presence of SIRS was not. Significantly higher median serum cortisol and lower median total thyroxine concentrations were seen in dogs with RA. Visually, urine was significantly darker and more turbid in dogs with RA, while USG, urinary pH, protein, bilirubin, and hemoglobin concentrations were not significantly different between both groups. All complications, except for IMHA and jaundice, occurred more frequently in dogs with RA. Survival at discharge was significantly lower in dogs with RA (Tables 3 and 4). 


\section{Discussion}

In our study, occurrence of RA at presentation was $14 \%$, demonstrating that RA is a relatively frequent event in dogs presenting with $B$. rossi infections. The hypothesis of a suspected underdiagnosis of RA in canine babesiosis, based on the traditional method of measuring urinary concentration (i.e., USG), was confirmed. By measuring urine osmolality, our results showed that USG led to an overestimation of the renal concentrating ability in many azotemic dogs with babesiosis.

The overwhelming majority of dogs with babesiosis presented to the OVAH are first opinion cases, making the study population a representative sample of the general population in the area around Onderstepoort. One study without apparent sampling bias (Lobetti and Jacobson, 2001), reported azotemia in $13 \%$ of the dogs presented with $B$. rossi, which is slightly lower but still comparable to the $17 \%$ azotemic dogs found in this study. Previous studies on B. rossi infection that made a clear distinction between prerenal and renal azotemia are not available. However, the most often cited reference diagnosed ARF in only 2.2\% (Jacobson and Clark, 1994), which was based on unpublished observations and without clearly defining ARF. This low number appears to be an underestimation, because the present study documented an almost 7 times higher occurrence of RA. Interestingly, the majority of azotemic dogs in our study (81\%) had RA (i.e., prerenal azotemia was diagnosed in only $19 \%$ of azotemic dogs). Therefore, when urine osmolality cannot be measured, the majority of azotemic dogs should be considered as suspected RA.

When USG was used to classify azotemia as prerenal or renal in origin, 71\% (15/21) of dogs with RA were misclassified as having prerenal azotemia, because of a systematic overestimation of urinary concentration by USG in our population of azotemic dogs with babesiosis (Fig. 1). These findings document that USG determination is not a reliable method to evaluate renal concentrating ability in this specific population. However, USG values below the cut-off of 1.030 were still clinically useful, because all azotemic dogs with a USG $<1.030$ were correctly identified to have RA. Presence of large molecules, such as hemoglobin, artefactually increases USG (Imran et al., 2010). This influence is clearly documented in human literature, where USG has been shown to overestimate urine osmolality in the presence of proteinuria and hemoglobinuria (Voinescu et al., 2002; Imran et al., 2010). To our knowledge, these interferences have not been reproduced in dogs. Proteinuria and hemoglobinuria did not significantly affect the correlation between USG and urine osmolality in 1 study in dogs (Ayoub et al., 2013). However, the small number of dogs with hemoglobinuria and the fact that the majority of dogs only had trace to moderate hemoglobinuria, could have limited the power to detect a significant effect of hemoglobinuria in that study (Ayoub et al., 2013). Although we suspect that the presence of large amounts of urinary hemoglobin and its degradation products are the main cause of the documented discrepancy between USG and urine osmolality in this population of azotemic dogs with babesiosis, this remains a hypothesis since we did not reliably quantify and correlate urinary hemoglobin. Urinary dipstick analysis did not show significant differences in hemoglobin concentrations between dogs with and without RA. However, since most urine samples had the maximum amount of hemoglobin concentration measurable by dipstick analysis $(4+)$, more exact quantification of urinary hemoglobin using a validated method is needed to reveal its role in the discrepancy between USG and urine osmolality, and to identify potential differences in urinary hemoglobin concentrations between dogs with and without RA. Our findings also raise questions regarding the reliability of USG measurements in other hemolytic diseases, such as IMHA.

Improved recognition of RA can help to optimize individual therapeutic decisions and can also help to assess prognosis. Individualized decisions regarding fluid therapy are essential, considering that ARDS is another devastating complication of babesiosis, in which unnecessary fluid therapy could be detrimental or even fatal. Our findings clearly indicate that concentrated urine based on USG should 
never be used as evidence of dehydration or appropriate renal concentrating ability in this specific population of azotemic dogs with babesiosis.

Several factors were associated with a diagnosis of RA. Although these statistical associations do not prove causality, they provide potentially interesting information regarding the pathogenesis of AKI in babesiosis and could help clinicians to differentiate between prerenal and renal azotemia. The Boerboel and Rottweiler are popular breeds in South Africa (Mellanby et al., 2011), yet they were proportionally overrepresented in the RA group, compared to the group without RA, which might suggest a breed susceptibility for kidney injury. Another study documented that the majority of B. canis-infected Rottweiler dogs also presented with complicated disease (Máthé et al., 2006). The higher body weight in dogs with RA could be explained by the overrepresentation of these 2 large breeds in the RA group. However, it is also possible that large breed dogs and dogs with a higher body weight had higher baseline serum creatinine values, making them more likely to have serum creatinine increased above the cut-off value used to define azotemia.

Increasing age was significantly associated with RA. Fig. 2 illustrates the presence of a population of older dogs with RA, while this subset of dogs is not represented in the group of dogs without RA. Acute renal failure was previously reported to be more common in older dogs with B. rossi infections (Jacobson and Clark, 1994). Older age was also associated with the risk of azotemia in a study with B. microti-likeinfected dogs (Camacho et al., 2004). Several studies in humans with malaria, a disease that shows many similarities with canine babesiosis (Reyers et al., 1998; Jacobson, 2006), documented that AKI is more common in adults than children (Mishra and Das, 2008; Wassmer et al., 2015). Pre-existing kidney disease or an age-related decrease in glomerular filtration rate could explain this finding (Cianciolo et al., 2016). Pre-existing CKD could lead to a higher susceptibility to develop AKI when challenged with babesiosis. Partly due to the retrospective nature of this study, it cannot be excluded that some dogs with pre-existing CKD were included. Especially dogs with early CKD, which are often without overt clinical signs, would likely not have been detected. However, this problem of potential pre-existing disease will be present in almost any clinical study including client-owned dogs. On the other hand, an age-dependent host response to the infection has been suggested before. One study hypothesized that older dogs with B. rossi infections seemed more likely to develop an acute overwhelming inflammatory response to the infection (Reyers et al., 1998). In humans with malaria and dogs with B. canis, older age was also a risk factor to develop multiple organ involvement (Máthé et al., 2006; Wassmer et al., 2015). Based on these data, it could be suggested that older dogs are more likely to develop AKI, not only because of possible pre-existing CKD, but potentially also because they would more strongly react to the infection with an acute overwhelming inflammatory response. This hypothesis of age-dependent host response has not directly been documented in canine babesiosis, but differences in host immune status and age-dependent changes in the vascular system response to infection-induced inflammation are also suggested in severe falciparum malaria (Wassmer et al., 2015).

Collapse at presentation was significantly more common in dogs with RA, which is consistent with several studies documenting collapse to be an important indicator of severe disease (Leisewitz et al., 2001; Böhm et al., 2006; Jacobson, 2006). Mean arterial pressure and presence of hypotension was not different between both groups. This contrasts to findings in B. canis-infected dogs, where significantly lower diastolic, systolic, and mean arterial pressures were seen in azotemic dogs (Zygner and Gójska-Zygner, 2014). It is possible that hypotension is not a major contributor to AKI in B. rossi, however presence of a type II error cannot be excluded.

The only hematological parameter that differed significantly between both groups was the band neutrophil count. The higher band neutrophil count in dogs with RA reinforces the hypothesis that SIRS is an important contributor to complications in babesiosis (Jacobson and Clark, 1994; Reyers et al., 1998; Welzl et al., 2001; Köster et al., 2015). The lack of a significant difference in the presence of SIRS 
between both groups is likely caused by the problematic application of SIRS criteria in this context (Okano et al., 2002). Three of the 4 SIRS criteria (rectal temperature, heart rate, and respiratory rate) could have been influenced by uremia and anemia. Anemia was not associated with RA, which is consistent with current literature (Zygner and Wedrychowicz, 2009).

Total bilirubin concentrations were significantly higher in dogs with RA, but severe hyperbilirubinemia (jaundice) was not significantly more common in dogs with RA. Research in malaria showed direct bilirubin concentrations and icterus to be independently associated with AKI (Saravu et al., 2014). Anemia caused by hemolysis (prehepatic icterus) is unlikely to be a major cause of the association between RA and total bilirubin, since anemia was not associated with RA. Hemolysis resulting in hemoglobinuric nephropathy is a controversial concept in canine babesiosis (Lobetti and Reyers, 1996). An experimental study was unable to document significant kidney injury after inducing severe hemoglobinemia (Lobetti et al., 1996). The conclusion was that hemoglobin by itself was not toxic to the kidney, but synergistic effects with other mediators of AKI could not be excluded. A recent study in malaria-induced AKI suggested that plasma cellfree hemoglobin and associated oxidative stress markers contribute to the pathogenesis of AKI (Plewes et al., 2017). Serum hemoglobin was significantly higher in dogs with RA in our study, but exact quantification of urinary hemoglobin is needed to further investigate its potential role in AKI. Hepatic dysfunction, leading to bile cast nephropathy and hepato-renal syndrome, is considered to contribute to AKI in malaria (Silva et al., 2017). It is possible that hepatic dysfunction is linked to hypoglycemia, because icterus has been associated with hypoglycemia in dogs with $B$. rossi before (Keller et al., 2004). It remains to be determined whether hepatic dysfunction (leading to a hepatic icterus) is a direct contributor to AKI in canine babesiosis. Lastly, acute pancreatitis, another more recently described complication of canine babesiosis (Jacobson, 2006; Köster et al., 2015), could also contribute to the development of jaundice (posthepatic icterus).

Higher serum cortisol and lower total thyroxine concentrations were seen in dogs with RA. These findings are consistent with studies in both babesiosis and parvovirus infection (Schoeman et al., 2007a; Schoeman et al., 2007b), that documented mortality and disease severity to be significantly associated with the same endocrine changes. These prognostic endocrine markers and RA are most likely not causally related, but rather associated because of an increased disease severity and mortality in this subset of dogs with babesiosis.

Urine was darker and more turbid in dogs with RA compared to dogs without RA. Hemoglobin or its degradation products are the most likely causes of dark and turbid urine in dogs with babesiosis. This is unlikely to be caused by bilirubinuria, since no difference between groups was found in urinary bilirubin concentrations. As discussed above, the role of hemoglobinuria in the development of RA in babesiosis remains speculative.

The presence of RA was associated with a worse outcome in this study. Multivariate analysis was not performed, because of the limited number of dogs in the group with RA. Hence, we could not establish RA as an independent variable in this study, inter alia because RA was significantly associated with most other complications, such as ARDS, cerebral babesiosis, and hypoglycemia, which could all influence outcome (Welzl et al., 2001; Nel et al., 2004). However, a previous study in dogs with B. rossi showed that the presence of azotemia, without clinical signs of dehydration, was associated with a higher risk of death compared to all other organ complications combined (Welzl et al., 2001).

Since urine osmolality is not readily available in most clinical situations and USG is unreliable in this specific population, identifying multiple factors such as older age, the presence of collapse, hyperphosphatemia, and more turbid or darker urine, could help clinicians to differentiate between prerenal and renal azotemia. Evaluation of these factors should always be combined with routine clinical parameters, such as the severity of azotemia and presence or absence of glucosuria, cylindruria, and renal tubular epithelial cells, indicative of AKI. 
Application of novel biomarkers of AKI, such as neutrophil gelatinase-associated lipocalin (Segev et al., 2013), could also be helpful in this context.

Since all data collections in this study were available from 3 previously performed prospective studies, many limitations inherent to retrospective studies were either absent or present to a lesser extent. Case inclusion and documentation of the medical records were standardized procedures during the previously performed prospective data collections, reducing inherent retrospective limitations. The use of different instruments (hematology and biochemistry analyzers, refractometers) throughout the study is a limitation inherent to the inclusion of data originating from 3 different cohorts. Although no sampling bias was present for the initial population of 297 dogs, urinalysis at admission was required for final inclusion in this study, which still could have led to both an under-or overestimation of RA in the final population of 152 dogs. In 2 of the cohorts (189/297 dogs), an attempt to collect urine at admission was made whenever a palpable urinary bladder was present, irrespective of the clinical condition of the dog. In the third cohort (108/297 dogs), urine collection was more likely to be performed in dogs that were hospitalized, potentially creating an overestimation of RA.

The occurrence of RA in $14 \%$ of the dogs was evaluated at admission only. It is highly likely that additional dogs have developed RA after presentation or during hospitalization. Importantly, RA includes dogs in advanced stages of AKI only. Because AKI represents a continuum of disease severity, ranging from very mild to severe kidney injury, our study design, which was based on a traditionally used cut-off value of urinary concentration to define RA, has obvious limitations and is unable to detect the majority of dogs with milder forms of AKI. Dogs with prerenal, fluid-responsive azotemia can also have AKI, but were categorized in the group of dogs without RA in this study. Lastly, dogs with mild azotemia might have been misclassified as non-azotemic because of hemolysis-induced underestimation of serum creatinine concentrations or an increased plasma volume after infection (Braun et al., 2003; de Scally et al., 2004; Schetters et al., 2009). Considering that $81 \%$ of azotemic dogs in this study had RA, and taking into account that dogs with prerenal azotemia can also sustain AKI, any dog that presents with azotemia should be considered to have AKI. Occurrence of RA in 14\% is clearly an underestimation of AKI in general, also because several studies have already documented that milder forms of AKI are very common in B. rossi infections (Lobetti and Jacobson, 2001; Defauw et al., 2012). But overall, identifying those dogs that are most severely affected (i.e., dogs with RA) has proven its worth in many clinical situations in order to optimize individual management.

Urinary sodium was not measured in this study. Dogs with prerenal azotemia but normal tubular function will retain sodium, leading to low urinary sodium concentrations. In contrast, dogs with AKI will have high urinary sodium concentrations due to tubular dysfunction (Waldrop, 2008). As a consequence, potential differences in urinary sodium concentrations between dogs with and without RA could have impacted the measurements of urine osmolality. Another limitation regarding the osmolality was its measurement after long-term storage of urine samples at $-80^{\circ} \mathrm{C}$. Samples were stored from 1 to 11 years before analysis. Although urine osmolality measured by freezing point depression was shown to remain stable in human urine stored at $-22^{\circ} \mathrm{C}$ for a very long time (at least 15 years) (Remer et al., 2014), no data on long-term stability are available in dogs. A recently published research study in dogs did document a minor decrease in urine osmolality during 90 days of storage at $-80^{\circ} \mathrm{C}$ (Reinhart et al., 2016). However, only 5 urine samples were evaluated in that study and the maximum change in urine osmolality over time was very small (less than 5\%). Hence, we cannot exclude that some dogs with a urine osmolality close to $1110 \mathrm{mOsmol} / \mathrm{kg}$ might have been misclassified. 


\section{Conclusion}

Our study demonstrated that RA is a relatively frequently occurring event in dogs with B. rossi infections. Moreover, RA was associated with a higher mortality. Furthermore, compared to the gold standard method of urinary concentration (i.e., urine osmolality), USG overestimated renal concentrating ability in the majority of azotemic dogs with babesiosis, which could lead to them being misclassified as having prerenal azotemia. Because severe hemoglobinuria is the most likely cause of the documented discrepancy between USG and urine osmolality, the reliability of USG to measure urinary concentration should also be considered and investigated in other hemolytic diseases.

\section{Conflict of interest statement}

Declarations of interest: none.

\section{Acknowledgements}

This study (cohort 3) was supported by a grant from the National Research Foundation of South Africa (grant number CPRR13080726333).

\section{References}

Ayoub, J.A., Beaufrere, H., Acierno, M.J., 2013. Association between urine osmolality and specific gravity in dogs and the effect of commonly measured urine solutes on that association. Am. J. Vet. Res. 74, 1542-1545.

Böhm, M., Leisewitz, A.L., Thompson, P.N., Schoeman, J.P., 2006. Capillary and venous Babesia canis rossi parasitaemias and their association with outcome of infection and circulatory compromise. Vet. Parasitol. 141, 18-29.

Braun, J.P., Lefebvre, H.P., Watson, A.D., 2003. Creatinine in the dog: a review. Vet. Clin. Pathol. 32, 162-179.

Camacho, A.T., Guitian, E.J., Pallas, E., Gestal, J.J., Olmeda, A.S., Goethert, H.K., Telford, S.R. 3rd, Spielman, A., 2004. Azotemia and mortality among Babesia microti-like infected dogs. J. Vet. Intern. Med. 18, 141-146.

Chadha, V., Garg, U., Alon, U.S., 2001. Measurement of urinary concentration: a critical appraisal of methodologies. Pediatr. Nephrol. 16, 374-382.

Cianciolo, R.E., Benali, S.L., Aresu, L., 2016. Aging in the canine kidney. Vet. Pathol. 53, 299-308.

Cowgill, L.D., Langston, C., 2011. Acute kidney insufficiency. In: Bartges, J., Polzin, D.J. (Eds.), Nephrology and Urology of Small Animals. Wiley-Blackwell, Chichester, pp. 472-523.

de Scally, M.P., Lobetti, R.G., Reyers, F., Humphris, D., 2004. Are urea and creatinine values reliable indicators of azotaemia in canine babesiosis? J. S. Afr. Vet. Assoc. 75, 121-124.

de Scally, M.P., Leisewitz, A.L., Lobetti, R.G., Thompson, P.N., 2006. The elevated serum urea:creatinine ratio in canine babesiosis in South Africa is not of renal origin. J. S. Afr. Vet. Assoc. 77, 175-178.

Defauw, P., Schoeman, J.P., Smets, P., Goddard, A., Meyer, E., Liebenberg, C., Daminet, S., 2012. Assessment of renal dysfunction using urinary markers in canine babesiosis caused by Babesia rossi. Vet. Parasitol. 190, 326-332. 
Furlanello, T., Fiorio, F., Caldin, M., Lubas, G., Solano-Gallego, L., 2005. Clinicopathological findings in naturally occurring cases of babesiosis caused by large form Babesia from dogs of northeastern Italy. Vet. Parasitol. 134, 77-85.

Glick, M.R., Ryder, K.W., Jackson, S.A., 1986. Graphical comparisons of interferences in clinical chemistry instrumentation. Clin. Chem. 32, $470-475$

Goddard, A., Leisewitz, A.L., Kristensen, A.T., Schoeman, J.P., 2015. Platelet indices in dogs with Babesia rossi infection. Vet. Clin. Pathol. 44, 493-497.

Goddard, A., Leisewitz, A.L., Kjelgaard-Hansen, M., Kristensen, A.T., Schoeman, J.P., 2016. Excessive pro-inflammatory serum cytokine concentrations in virulent canine babesiosis. PLoS ONE 11, e0150113.

Imran, S., Eva, G., Christopher, S., Flynn, E., Henner, D., 2010. Is specific gravity a good estimate of urine osmolality? J. Clin. Lab. Anal. 24, $426-430$

Irwin, P.J., 2010. Canine babesiosis. Vet. Clin. North Am. Small Anim. Pract. 40, 1141-1156.

Jacobson, L.S., 2006. The South African form of severe and complicated canine babesiosis: clinical advances 1994-2004. Vet. Parasitol. 138, 126-139.

Jacobson, L.S., Clark, I.A., 1994. The pathophysiology of canine babesiosis: new approaches to an old puzzle. J. S. Afr. Vet. Assoc. 65, 134145.

Keller, N., Jacobson, L.S., Nel, M., de Clerq, M., Thompson, P.N., Schoeman, J.P., 2004. Prevalence and risk factors of hypoglycemia in virulent canine babesiosis. J. Vet. Intern. Med. 18, 265-270.

Köster, L.S., Steiner, J.M., Suchodolski, J.S., Schoeman, J.P., 2015. Serum canine pancreatic-specific lipase concentrations in dogs with naturally occurring Babesia rossi infection. J. S. Afr. Vet. Assoc. 86, E1-E7.

Leisewitz, A.L., Jacobson, L.S., de Morais, H.S., Reyers, F., 2001. The mixed acid-base disturbances of severe canine babesiosis. J. Vet. Intern. Med. 15, 445-452.

Lobetti, R.G., Reyers, F., 1996. Met-haemoglobinuria in naturally occurring Babesia canis infection. J. S. Afr. Vet. Assoc. 67, 88-90.

Lobetti, R.G., Jacobson, L.S., 2001. Renal involvement in dogs with babesiosis. J. S. Afr. Vet. Assoc. 72, 23-28.

Lobetti, R.G., Reyers, F., Nesbit, J.W., 1996. The comparative role of haemoglobinaemia and hypoxia in the development of canine babesial nephropathy. J. S. Afr. Vet. Assoc. 67, 188-198.

Máthé, Á., Vörös, K., Papp, L., Reiczigel, J., 2006. Clinical manifestations of canine babesiosis in Hungary (63 cases). Acta Vet. Hung. 54, 367385.

Matjila, P.T., Penzhorn, B.L., Bekker, C.P., Nijhof, A.M., Jongejan, F., 2004. Confirmation of occurrence of Babesia canis vogeli in domestic dogs in South Africa. Vet. Parasitol. 122, 119-125.

Mellanby, R.J., Handel, I.G., Clements, D.N., Bronsvoort, B.M., Lengeling, A., Schoeman, J.P., 2011. Breed and sex risk factors for canine babesiosis in South Africa. J. Vet. Intern. Med. 25, 1186-1189.

Mishra, S.K., Das, B.S., 2008. Malaria and acute kidney injury. Semin. Nephrol. 28, 395-408.

Nel, M., Lobetti, R.G., Keller, N., Thompson, P.N., 2004. Prognostic value of blood lactate, blood glucose, and hematocrit in canine babesiosis. J. Vet. Intern. Med. 18, 471-476. 
Okano, S., Yoshida, M., Fukushima, U., Higuchi, S., Takase, K., Hagio, M., 2002. Usefulness of systemic inflammatory response syndrome criteria as an index for prognosis judgement. Vet. Rec. 150, 245-246.

Plewes, K., Kingston, H.W.F., Ghose, A., Maude, R.J., Herdman, M.T., Leopold, S.J., Ishioka, H., Hasan, M.M.U., Haider, M.S., Alam, S., Piera, K.A., Charunwatthana, P., Silamut, K., Yeo, T.W., Faiz, M.A., Lee, S.J., Mukaka, M., Turner, G.D.H., Anstey, N.M., Roberts, L.J. 2nd, White, N.J., Day, N.P.J., Hossain, M.A., Dondorp, A.M., 2017. Cell-free hemoglobin mediated oxidative stress is associated with acute kidney injury and renal replacement therapy in severe falciparum malaria: an observational study. BMC Infect. Dis. 17, 313.

Rees, P., Schoeman, J.P., 2008. Plasma insulin concentrations in hypoglycaemic dogs with Babesia canis rossi infection. Vet. Parasitol. 152, 60-66.

Reinhart, J.M., White, B.J., Pohlman, L.M., Schermerhorn, T., 2016. Stability of osmolality in previously frozen canine serum and urine samples. Vet. Clin. Pathol. 45, 665-668.

Remer, T., Montenegro-Bethancourt, G., Shi, L., 2014. Long-term urine biobanking: storage stability of clinical chemical parameters under moderate freezing conditions without use of preservatives. Clin. Biochem. 47, 307-311.

Reyers, F., Leisewitz, A.L., Lobetti, R.G., Milner, R.J., Jacobson, L.S., van Zyl, M., 1998. Canine babesiosis in South Africa: more than one disease. Does this serve as a model for falciparum malaria? Ann. Trop. Med. Parasitol. 92, 503-511.

Ruiz de Gopegui, R., Peñalba, B., Goicoa, A., Espada, Y., Fidalgo, L.E., Espino, L., 2007. Clinico-pathological findings and coagulation disorders in 45 cases of canine babesiosis in Spain. Vet. J. 174, 129-132.

Saravu, K., Rishikesh, K., Parikh, C.R., 2014. Risk factors and outcomes stratified by severity of acute kidney injury in malaria. PLoS ONE 9, e90419.

Schetters, T.P., Kleuskens, J.A., Van De Crommert, J., De Leeuw, P.W., Finizio, A.L., Gorenflot, A., 2009. Systemic inflammatory responses in dogs experimentally infected with Babesia canis; a haematological study. Vet. Parasitol. 162, 7-15.

Schoeman, J.P., Herrtage, M.E., 2007. The response of the pituitary-adrenal and pituitary-thyroidal axes to the plasma glucose perturbations in Babesia canis rossi babesiosis. J. S. Afr. Vet. Assoc. 78, 215-220.

Schoeman, J.P., Herrtage, M.E., 2008. Adrenal response to the low dose ACTH stimulation test and the cortisol-to-adrenocorticotrophic hormone ratio in canine babesiosis. Vet. Parasitol. 154, 205-213.

Schoeman, J.P., Goddard, A., Herrtage, M.E., 2007a. Serum cortisol and thyroxine concentrations as predictors of death in critically ill puppies with parvoviral diarrhea. J. Am. Vet. Med. Assoc. 231, 1534-1539.

Schoeman, J.P., Rees, P., Herrtage, M.E., 2007b. Endocrine predictors of mortality in canine babesiosis caused by Babesia canis rossi. Vet. Parasitol. 148, 75-82.

Segev, G., Palm, C., LeRoy, B., Cowgill, L.D., Westropp, J.L., 2013. Evaluation of neutrophil gelatinase-associated lipocalin as a marker of kidney injury in dogs. J. Vet. Intern. Med. 27, 1362-1367.

Silva, G.B.D.J., Pinto, J.R., Barros, E.J.G., Farias, G.M.N., Daher, E.F., 2017. Kidney involvement in malaria: an update. Rev. Inst. Med. Trop. Sao Paulo 59, e53.

Voinescu, G.C., Shoemaker, M., Moore, H., Khanna, R., Nolph, K.D., 2002. The relationship between urine osmolality and specific gravity. Am. J. Med. Sci. 323, 39-42.

Waldrop, J.E., 2008. Urinary electrolytes, solutes, and osmolality. Vet. Clin. North Am. Small Anim. Pract. 38, 503-512. 
Wassmer, S.C., Taylor, T.E., Rathod, P.K., Mishra, S.K., Mohanty, S., Arevalo-Herrera, M., Duraisingh, M.T., Smith, J.D., 2015. Investigating the pathogenesis of severe malaria: a multidisciplinary and cross-geographical approach. Am. J. Trop. Med. Hyg. 93, 42-56.

Welzl, C., Leisewitz, A.L., Jacobson, L.S., Vaughan-Scott, T., Myburgh, E., 2001. Systemic inflammatory response syndrome and multipleorgan damage/dysfunction in complicated canine babesiosis. J. S. Afr. Vet. Assoc. 72, 158-162.

WHO, 2014. Severe malaria. Trop. Med. Int. Health 19 (Suppl 1), S7-S131.

Zygner, W., Wedrychowicz, H., 2009. Influence of anaemia on azotaemia in dogs infected with Babesia canis in Poland. Bull. Vet. Inst. Pulawy $53,663-668$

Zygner, W., Gójska-Zygner, O., 2014. Association between decreased blood pressure and azotaemia in canine babesiosis. Pol. J. Vet. Sci. 17, $173-175$ 


\section{Table 1}

Clinical data from dogs with renal azotemia and dogs without renal azotemia at presentation. All variables are continuous and expressed as median (range). Unless specified otherwise, data were available for all dogs.

\begin{tabular}{llll}
\hline Variable & RA $(\mathrm{n}=21)$ & No RA $(\mathrm{n}=131)$ & $P$-value \\
\hline Age (months) & $37(8-120)$ & $19(3-144)$ & 0.016 \\
Body weight $(\mathrm{kg})$ & $30.6(9.8-65)(\mathrm{n}=19)$ & $14.5(3-55)(\mathrm{n}=124)$ & $<0.001$ \\
Duration of illness (days) & $2(1-7)(\mathrm{n}=19)$ & $3(1-21)(\mathrm{n}=121)$ & 0.14 \\
Body temperature $\left({ }^{\circ} \mathrm{C}\right)$ & $39.0(<32.0-40.2)$ & $39.6(32.9-41.2)$ & 0.036 \\
Heart rate (beats/min) & $130(24-160)$ & $132(28->200)(\mathrm{n}=130)$ & 0.30 \\
RR (breaths/min) & $41(9-84)(\mathrm{n}=20)$ & $48(15-128)(\mathrm{n}=121)$ & 0.46 \\
MAP (mmHg) & $117(67-139)(\mathrm{n}=14)$ & $105(68-201)(\mathrm{n}=79)$ & 0.59 \\
\hline
\end{tabular}

RA: renal azotemia.

RR: respiratory rate.

MAP: mean arterial pressure. 


\section{Table 2}

Clinicopathological findings from dogs with renal azotemia and dogs without renal azotemia at presentation. All variables are continuous and expressed as median (range). Unless specified otherwise, data were available for all dogs.

\begin{tabular}{|c|c|c|c|c|}
\hline Variable (unit) & RI & RA $(n=21)$ & No RA $(n=131)$ & $P$-value \\
\hline Hematocrit & $0.37-0.55$ & $0.21(0.05-0.60)$ & $0.16(0.04-0.53)$ & 0.31 \\
\hline $\mathrm{WBC}\left(\mathrm{x} 10^{9} / \mathrm{L}\right)$ & $6-15$ & $7.7(1.1-104)$ & $6.7(1.1-33.1)$ & 0.14 \\
\hline Segmented neutrophil count ( $\left.\times 10^{9} / \mathrm{L}\right)$ & $3-11.5$ & $4.8(0.04-67.3)$ & $4.4(0.7-20.5)$ & 0.38 \\
\hline Band neutrophil count (x $\left.10^{9} / \mathrm{L}\right)$ & $0-0.5$ & $1.1(0-21.8)$ & $0.5(0-9.1)$ & 0.013 \\
\hline Platelet count $\left(\times 10^{9} / \mathrm{L}\right)$ & $200-500$ & $25(0-147)$ & $27(0-636)$ & 0.90 \\
\hline Serum creatinine $(\mu \mathrm{mol} / \mathrm{L})$ (cohort 1$)$ & $40-133$ & $203(142-527)(n=7)$ & $72(41-298)(n=35)$ & \\
\hline Serum creatinine $(\mu \mathrm{mol} / \mathrm{L})($ cohort 2,3$)$ & 59-109 & $161(125-865)(n=14)$ & $59(23-131)(n=96)$ & \\
\hline Serum urea $(\mathrm{mmol} / \mathrm{L})$ & $2.3-8.9$ & $28.4(8.9-104)(n=15)$ & $8.2(3.0-34.6)(n=89)$ & $<0.001$ \\
\hline Glucose (mmol/L) & $3.3-5.5$ & $4.2(0.4-9.9)$ & $4.6(0.5-22.2)(n=130)$ & 0.045 \\
\hline Total bilirubin $(\mu \mathrm{mol} / \mathrm{L})$ & $1.0-6.8$ & $42.5(5.5-465)(n=13)$ & $9.8(0.4-408)(n=101)$ & 0.039 \\
\hline Cortisol (nmol/L) & $10-160$ & $425(67-690)(n=12)$ & $122(<5.5-861)(n=73)$ & 0.0018 \\
\hline Total thyroxine (nmol/L) & $15-45$ & $3.3(<1.16-15.1)(n=12)$ & $11.9(<1.16-34.1)(n=74)$ & 0.0048 \\
\hline Blood lactate (mmol/L) & $\leq 2.5$ & $5.9(1.3-13.2)(n=13)$ & $2.8(<0.8-23.9)(\mathrm{n}=91)$ & 0.026 \\
\hline Serum hemoglobin $(\mathrm{g} / \mathrm{L})$ & & $0.5(0-1.3)(n=7)$ & $0(0-0.6)(n=35)$ & 0.0026 \\
\hline Urinary $\mathrm{pH}$ & & $6(5-7)(n=19)$ & $6(5-8)(n=128)$ & 0.057 \\
\hline Urine specific gravity & & $1.036(1.014->1.050)$ & $1.040(1.003->1.050)(\mathrm{n}=129)$ & 0.37 \\
\hline Urine osmolality (mOsmol/kg) & & $708(397-1092)$ & $1090(204-2665)(n=127)$ & \\
\hline
\end{tabular}

RI: reference interval.

RA: renal azotemia.

WBC: white blood cell count.

Cohort 1: data collections from 2006. Cohort 2: data collections from 2011-2013. Cohort 3: data collections from 2013-2015. 


\section{Table 3}

Urinalysis results from dogs with renal azotemia and dogs without renal azotemia at presentation.

1

\begin{tabular}{|c|c|c|c|}
\hline Variable & RA & no RA & P-value \\
\hline $\begin{array}{l}\text { Urinary protein } \\
\text { nat }\end{array}$ & & & 0.78 \\
\hline 0 & $1 / 20(5 \%)$ & $10 / 128(8 \%)$ & \\
\hline $1+$ & $4 / 20(20 \%)$ & $22 / 128(17 \%)$ & \\
\hline $2+$ & $3 / 20(15 \%)$ & $24 / 128(19 \%)$ & \\
\hline $3+$ & $12 / 20(60 \%)$ & $72 / 128(56 \%)$ & \\
\hline Urinary bilirubin & & & 0.81 \\
\hline 0 & $3 / 20(15 \%)$ & $13 / 127(10 \%)$ & \\
\hline $1+$ & $2 / 20(10 \%)$ & $15 / 127(12 \%)$ & \\
\hline $2+$ & $4 / 20(20 \%)$ & $38 / 127(30 \%)$ & \\
\hline $3+$ & $11 / 20(55 \%)$ & $61 / 127(48 \%)$ & \\
\hline Urinary blood/hemoglobin & & & 0.052 \\
\hline 0 & $0 / 20(0 \%)$ & $15 / 124(12 \%)$ & \\
\hline $1+$ & $0 / 20(0 \%)$ & $6 / 124(5 \%)$ & \\
\hline $2+$ & $0 / 20(0 \%)$ & $7 / 124(6 \%)$ & \\
\hline $3+$ & $3 / 20(15 \%)$ & $14 / 124(11 \%)$ & \\
\hline $4+$ & $17 / 20(85 \%)$ & $82 / 124(66 \%)$ & \\
\hline Urine color & & & 0.0086 \\
\hline Light yellow & $0 / 19(0 \%)$ & $5 / 129(4 \%)$ & \\
\hline (Dark) yellow & $6 / 19(32 \%)$ & $70 / 129(54 \%)$ & \\
\hline Amber / red & $1 / 19(5 \%)$ & $21 / 129(16 \%)$ & \\
\hline Dark red / brown & $10 / 19(53 \%)$ & $23 / 129(18 \%)$ & \\
\hline Dark brown / black & $2 / 19(10 \%)$ & $10 / 129(8 \%)$ & \\
\hline Urine turbidity & & & $<0.001$ \\
\hline Clear or slightly turbid & $0 / 11(0 \%)$ & $76 / 111(68 \%)$ & \\
\hline Moderate or severely turbid & $11 / 11(100 \%)$ & $35 / 111(32 \%)$ & \\
\hline
\end{tabular}

RA: renal azotemia. 


\section{Table 4}

Binary and ordinal data from dogs with renal azotemia and dogs without renal azotemia at presentation.

\begin{tabular}{|c|c|c|c|}
\hline Variable & RA & no RA & P-value \\
\hline Collapsed & $11 / 21(52 \%)$ & $29 / 128(23 \%)$ & 0.0074 \\
\hline Hypotensive (MAP $<80 \mathrm{mmHg}$ ) & $2 / 14(14 \%)$ & $5 / 79(6 \%)$ & 0.28 \\
\hline Presence of severe anemia (hematocrit $<0.15$ ) & $9 / 21(43 \%)$ & $55 / 131(42 \%)$ & 1.0 \\
\hline Absence of anemia (hematocrit $\geq 0.37$ ) & $5 / 21(24 \%)$ & $15 / 131(11 \%)$ & 0.16 \\
\hline Presence of SIRS & $17 / 21(81 \%)$ & $89 / 124(72 \%)$ & 0.44 \\
\hline Hyperphosphatemia & $12 / 13(92 \%)$ & $16 / 81(20 \%)$ & $<0.001$ \\
\hline Hyperlactatemia (blood lactate $>2.5 \mathrm{mmol} / \mathrm{L}$ ) & $9 / 13(69 \%)$ & $50 / 91(55 \%)$ & 0.38 \\
\hline In-saline agglutination test positive & $2 / 20(10 \%)$ & $22 / 129(17 \%)$ & 0.53 \\
\hline Hemoconcentration & $4 / 21(19 \%)$ & $4 / 131(3 \%)$ & 0.013 \\
\hline Jaundice & $6 / 13(46 \%)$ & $20 / 101(20 \%)$ & 0.071 \\
\hline \multicolumn{3}{|l|}{ Hypoglycemia } & 0.0022 \\
\hline Absent (glucose $\geq 3.3 \mathrm{mmol} / \mathrm{L}$ ) & $11 / 21(52 \%)$ & $106 / 130(82 \%)$ & \\
\hline \multicolumn{2}{|c|}{ Mild (glucose $<3.3 \mathrm{mmol} / \mathrm{L},>2.2 \mathrm{mmol} / \mathrm{L}) 4 / 21(19 \%)$} & $13 / 130(10 \%)$ & \\
\hline Severe (glucose $<2.2 \mathrm{mmol} / \mathrm{L}$ ) & $6 / 21(29 \%)$ & $11 / 130(8 \%)$ & \\
\hline Cerebral babesiosis & $4 / 21(19 \%)$ & $1 / 131(1 \%)$ & 0.0013 \\
\hline ARDS & $6 / 21(29 \%)$ & $3 / 131(2 \%)$ & $<0.001$ \\
\hline Presence of $\geq 1$ other complication & $20 / 21(95 \%)$ & $82 / 131(63 \%)$ & 0.0022 \\
\hline Outcome at discharge (death/euthanasia) & $12 / 21(57 \%)$ & $8 / 129(6 \%)$ & $<0.001$ \\
\hline
\end{tabular}

RA: renal azotemia.

MAP: mean arterial pressure.

SIRS: systemic inflammatory response syndrome.

ARDS: acute respiratory distress syndrome. 
Figures

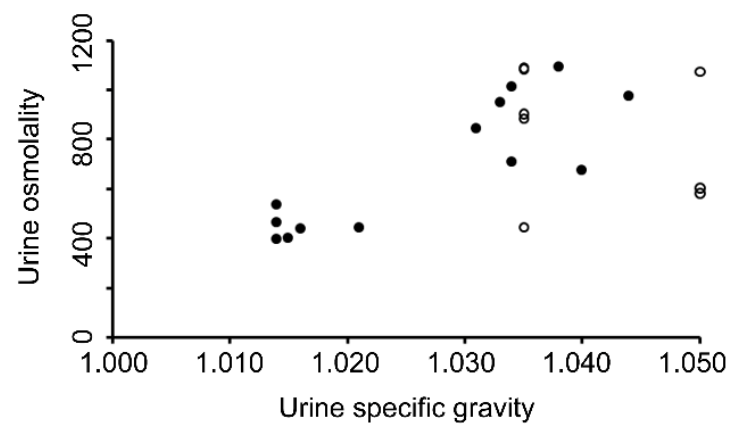

Fig. 1. Scatterplot of urine specific gravity versus urine osmolality in dogs with renal azotemia $(\mathrm{n}=21)$. Data points from all individual dogs are visualized as black dots and circles. The black dots represent dogs with an exact USG measurement value, while the circles represent dogs with USG values higher than the highest calibrated point on the specific gravity scale of each refractometer used (USG $>1.035$ and $U S G>1.050$, respectively). 

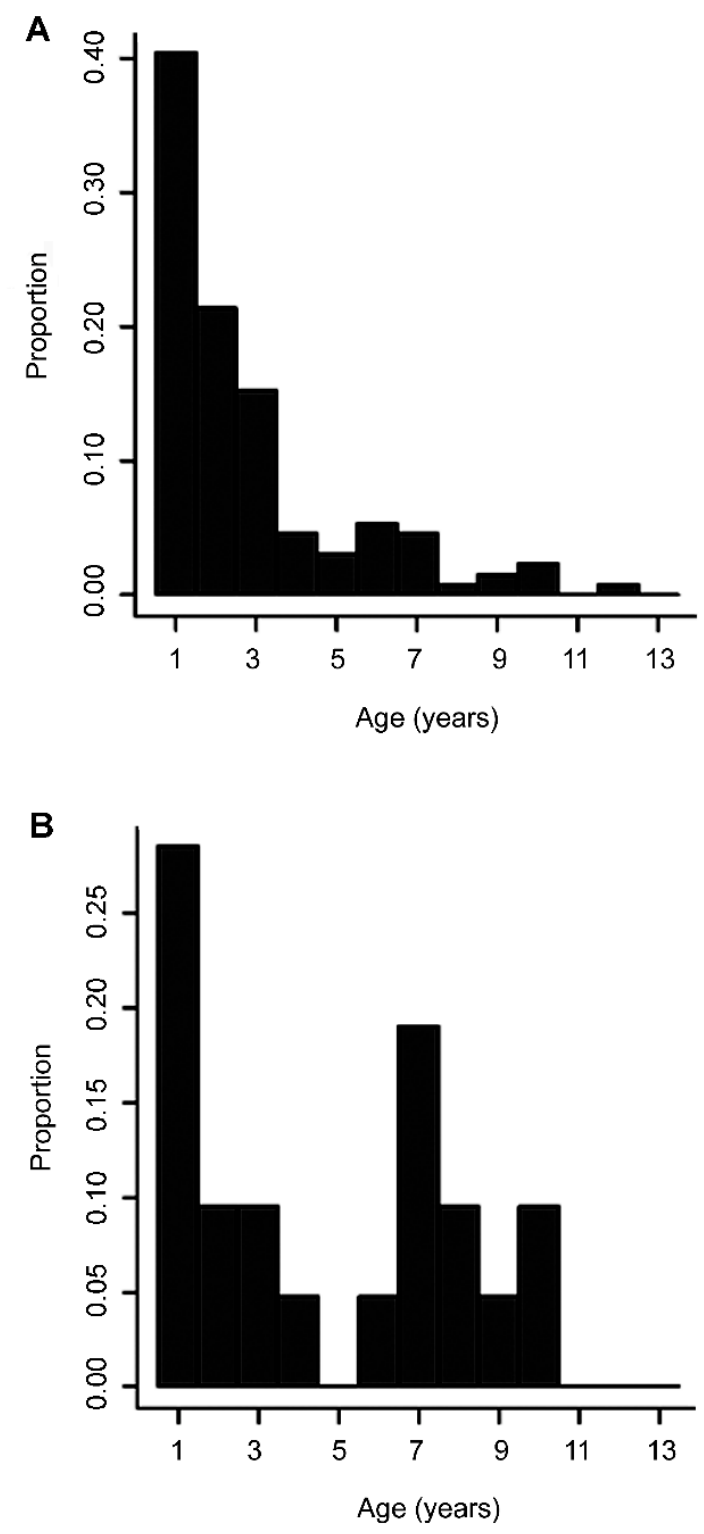

Fig. 2. Age distribution of dogs without renal azotemia $(\mathbf{A}, \mathrm{n}=131)$ and dogs with renal azotemia $(\mathbf{B}, \mathrm{n}=21)$. 